\title{
Théorie(s) de la fabrication des espaces
}

Theory/Theories on the Making of Cities

\section{Guy Tapie}

\section{OpenEdition}

\section{Journals}

Édition électronique

URL : http://journals.openedition.org/crau/573

DOI : $10.4000 /$ crau. 573

ISSN : 2547-5746

\section{Éditeur}

Éditions du patrimoine

\section{Édition imprimée}

Date de publication : 1 novembre 2012

Pagination : 172-181

ISBN : 978-2-7577-0108-9

ISSN : 1296-4077

Référence électronique

Guy Tapie, "Théorie(s) de la fabrication des espaces », Les Cahiers de la recherche architecturale et urbaine [En ligne], 26/27 | 2012, mis en ligne le 01 novembre 2017, consulté le 03 mai 2019. URL : http://journals.openedition.org/crau/573 ; DOI : 10.4000/crau.573 
La volonté de qualifier la façon dont se fabriquent les espaces contemporains quelle que soit l'échelle concernée (l'architecture, la ville, le territoire) n'est pas nouvelle pour la recherche architecturale et urbaine contemporaine. Elle en a fait un thème de théorisation et d'investigation pour interpréter les transformations des architectes et leurs conditions d'action, alternant entre une approche sur la profession ou les métiers et une autre sur le projet en considérant sa fabrication collective. Ces perspectives ouvrent vers une réflexion critique sur le rôle de tels professionnels dans les sociétés contemporaines, au moment d'une transformation assez radicale dans tous les domaines de la fabrication des espaces bâtis.

\section{Théorie(s) de la fabrication des espaces}

GUY TAPIE

La volonté de qualifier la façon dont se fabriquent les espaces contemporains quelle que soit l'échelle concernée (l'architecture, la ville, le territoire) n'est pas nouvelle pour la recherche architecturale et urbaine contemporaine qui en a fait un thème pertinent d'investigation. Cela s'est fait non sans ambiguïté, ni sans critique : le processus de production n'efface-t-il pas l'objet bâti ? Tels des vases communicants, en s'intéressant aux acteurs et aux dispositifs, beaucoup ont cru perdre de vue l'essentiel, l'espace conçu, les formes, voire l'usage, en un mot l'architecture. Pourtant quand la sociologie de groupes professionnels décrypte les pratiques des policiers, des avocats, des journalistes, des enseignants, des managers et des "traders", elle aborde la conception de la sécurité et des rapports à la loi, la production et le contrôle de l'information, les transformations de l'éducation, l'organisation des sanctuaires de l'économie moderne. Quand les sciences politiques et de gestion analysent les modes de gouvernement et d'organisation, elles abordent les mécanismes de pouvoir et de légitimation politique, le statut des 
citoyens, ou l'efficacité du management. Acteurs et action sociale s'entremêlent pour engendrer les règles de la vie collective, même si la recherche d'intelligibilité des processus conduit à les séparer comme l'ont opéré les théories sur la fabrication de l'espace.

L'analyse des acteurs de la production architecturale et urbaine et des architectes en particulier n'échappe pas à ce postulat. Les professionnels préfigurent et formatent des dispositifs spatiaux qui soudent et expriment les relations entre individus ou entre groupes : habiter, se détendre, se déplacer, travailler, apprendre, se soigner, faire de la politique, administrer. Ils anticipent et réalisent, plus que le décor, la scène où se jouent les rapports sociaux dans une symbiose souvent mise en évidence entre une société et son espace bâti. Le postulat de l'encastrement des stratégies des architectes au contexte sociétal justifie l'intérêt pour leurs modalités d'intervention, leurs modes d'organisation et leur identité professionnelle et sociale. Historiquement, au service d'une autorité supérieure - religieuse, politique, sociale, économique - quelques démiurges projetaient en d'audacieuses constructions le pouvoir qui s'imposait à tous ou de façon moins spectaculaire, couvraient le territoire de signes d'une appartenance collective. L'architecture monumentale en est l'expression universelle et continue à émettre des signaux forts aux populations par la création des lieux de la post-modernité où se mêlent fréquemment édifices religieux, monuments dédiés aux pouvoirs politiques, et temples de la finance ou de la consommation.

Plus précisément, par la fabrication d'un espace public, il est question de la représentation de la démocra- tie, du vivre ensemble, du lien social ; par l'habitat, un projet personnel, familial, social, est mis en forme; par l'aménagement du territoire et les grandes infrastructures, une nation s'équipe; par la transformation de la ville, de nouvelles cultures des lieux se manifestent, entre mondialisation et localisation. À l'évidence, les formes et l'organisation de l'espace échappent de moins en moins à l'action des architectes même si, à juste titre, des chercheurs soulignent l'importance d'une architecture auto-construite (le vernaculaire, l'informel, le spontané) ou le rôle évident d'autres acteurs (entrepreneurs, promoteurs, ingénieurs, autres experts). Dans les sociétés développées contemporaines, la division du travail crée un espace professionnel original et complexe marqué par la tradition des corporations et des formes d'organisation capitalistiques. Ceux qui participent à l'élaboration des espaces ont alors un rôle dont l'autonomie est interrogée : opérateurs diaphanes, c'est un principe social supérieur qui explique l'usage et de la production spatiale (l'État, les classes dirigeantes, une puissance divine, la communauté, la société, la culture) ; médiateurs puissants d'aspirations sociales, c'est leur autonomie et leur force qui sont soulignées pour figurer ou préfigurer les espaces contemporains. Au travers d'une analyse du rôle de ces professionnels, sont en jeu les capacités des démocraties à générer des corps d'experts indépendants des pouvoirs tels qu'on les caractérisait au moment de l'organisation des États modernes et démocratiques.

De nombreux chercheurs se sont focalisés sur le rôle des architectes au prisme d'investigations sur la conception, en tenant compte d'une conviction partagée par 


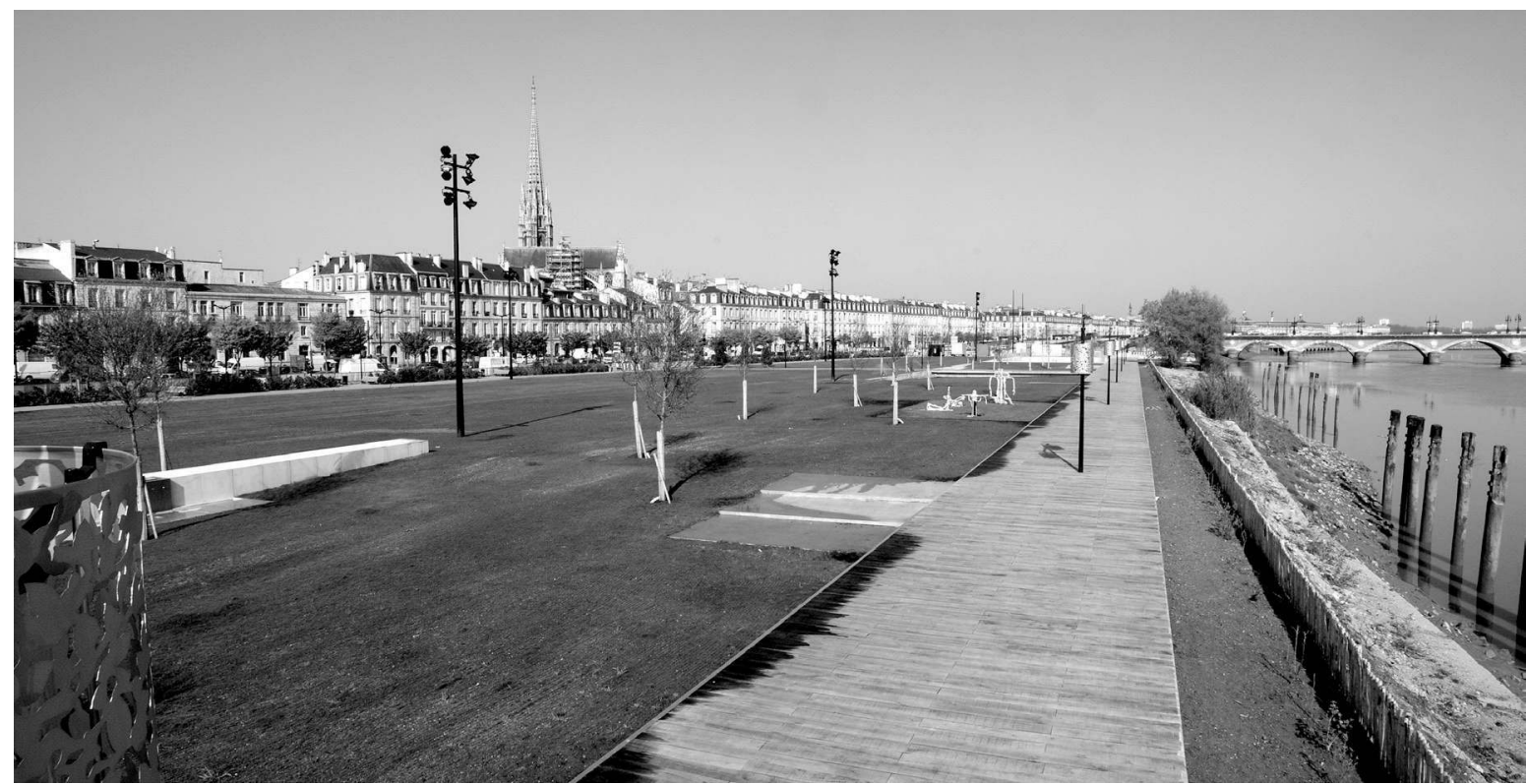

\section{Aménagement}

des quais de Bordeaux, 1999- 2009.

Photo G. T.

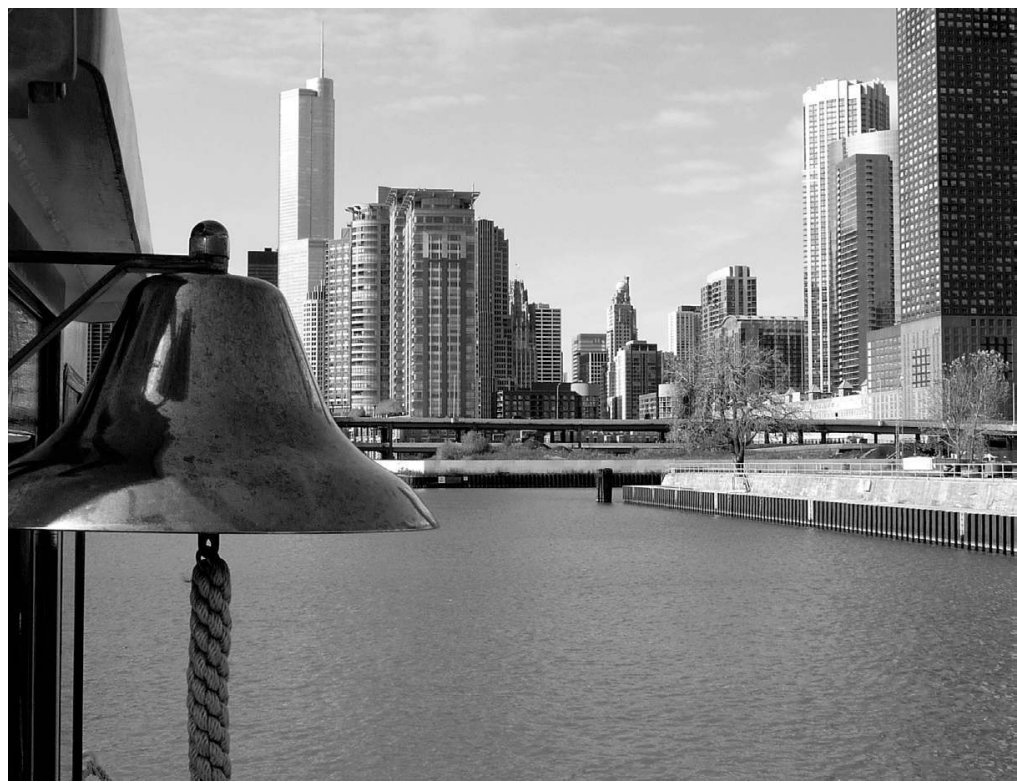

Chicago, quartier

d'affaires le Loop, 2009.

Photo G. T. 
le milieu (professionnels, associations professionnelles, administrations en charge de l'architecture) sur la singularité des problèmes d'architecture. Position qui s'appuie sur deux arguments principaux : d'abord, la nature culturelle et symbolique de toute construction conduit à affirmer que l'objet bâti n'est pas un banal produit de consommation, car il incorpore des capacités d'identification individuelle et sociale; ensuite, l'originalité de son mode de fabrication fait la part belle à l'auteur, au créateur, à la conception. De fait, l'essence du travail des architectes, la conception et le projet, est apparue comme un objet légitime et exclusif de recherche. Pour débattre des concepts, des doctrines, des choix projectuels, pour retracer l'histoire des mouvements de pensée et caractériser les courants contemporains, un champ de connaissances s'est constitué. Recherche qui oscille entre journalisme critique, médiation de l'architecture et benchmarking pour faire connaître les bonnes pratiques conceptuelles et projectuelles. Des versions plus scientifiques, I'architecturologie par exemple, les traités de composition spatiale, I'histoire des mouvements architecturaux, se sont démarqués de discours auto-justificateurs pour objectiver l'un des aspects centraux de la production architecturale, I'activité créative, et pour resituer dans l'histoire les formes projetées ou construites, les doctrines et les contextes de leur fabrication. Ainsi la réflexion sur la conception et sur le projet, soutenue par l'histoire, ferait l'originalité d'une discipline architecturale non réductible aux approches des sciences humaines sur l'architecture, des sciences de l'ingénieur sur l'objet construit ou des disciplines de l'art sur l'esthétique.
L'attention accordée aux formes de représentation graphique, du croquis à main levée à la sophistication des représentations informatiques, comme éléments syncrétiques d'une pensée est une des clés revendiquée de la conception. L'interprétation des intentions des auteurs est aussi fouillée pour identifier les doctrines. Le travail de chercheurs, dont la ligne directrice est la fabrication des espaces, a montré combien on ne pouvait pas la réduire à la seule analyse substantialiste du projet et de la conception.

\section{Professions, métiers : la diffusion de cultures professionnelles}

Nous avons déjà mis en évidence lors des deux dernières décennies les changements en œuvre chez les architectes autour du triptyque : professions, compétences et services. Les recherches effectuées montrent la capacité des architectes à jouer de tous les mouvements des sociétés, des marchés, des dispositifs de production, et à pondérer les thèses de la déprofessionnalisation et celle du déclin annoncé depuis les années 1970. Une dispersion de leurs activités, la constitution de figures de compétences démultipliant un savoir, la mutuelle dépendance d'autres acteurs réduisent leur pouvoir si l'on se fie à quelques représentations nostalgiques. L'adaptation des architectes à leurs contextes de travail se déploie sur plusieurs registres, à la croisée des contraintes des marchés et des processus de production.

Ainsi sur des registres assez similaires, les marchés de l'immobilier de bureaux, le secteur de la maison individuelle, ou l'intervention dans l'existant, ont mis 
en exergue le besoin " de service " de la part des clients amenant l'architecte à valoriser le prix, l'économie, la concrétisation du projet, les délais, I'adaptation fonctionnelle, quelquefois une esthétique originale, image contemporaine pour être en accord avec l'air du temps. Néanmoins, le prix de revient au mètre carré est un leitmotiv qui s'affiche à la manière d'un audimat, indice probant d'efficacité, principal credo conceptuel. L'exercice d'architecture orienté par une finalité économique doit savoir conjuguer des ambitions esthétiques et fonctionnelles. Avec prudence, l'évangélisation architecturale de profanes difficiles à convertir propage des démarches de qualité et montre aux clients l'intérêt à bonifier à moindres coûts leur stratégie immobilière par un travail sur l'architecture. De tels marchés ne sont pas autistes à un discours doctrinal, indispensable pour les architectes afin de rester dans le champ architectural. L'intellectualisation de la prestation, même si on ne l'ébruite pas au risque de heurter les clients, reste un moyen de distinction puissant par rapport aux autres concurrents, promoteurs principalement, et vis-à-vis des métiers techniques.

Pour répondre aux exigences de chaque segment de marché, les architectes se spécialisent donc en adoptant les valeurs et les comportements en œuvre dans chacun. L'un des effets de cette socialisation préférentielle redoutée par les architectes est la réclusion perpétuelle dans un segment de marché ou un type d'intervention. Cette relégation est, non seulement symboliquement infamante quand les pairs, la presse spécialisée, n'y lisent pas une intention ou un acte d'architecture. Surtout, il y a la peur de ne pouvoir se mouvoir vers d'autres activités. Pourtant la carrière des architectes se joue de moins en moins dans un univers confiné en raison de la dynamique des marchés ; l'immobilier de bureaux a suivi la courbe fluctuante de l'économie des pays développés; les marchés de l'existant, limités dans un premier temps à la remise en état, sont devenus un marché honorable en termes de conception ; la maison individuelle est en attente d'alternatives face à la banalisation pavillonnaire et à une demande plus exigeante. Des carrières démarrées sous le joug de la simple remise en état dans l'existant, sous l'autorité omnipotente de promoteurs peu sensibles à la valeur architecturale dans le tertiaire ou l'industriel, par la réalisation de quelques maisons individuelles, peuvent aboutir à des commandes plus emblématiques, plus crédibles en termes doctrinaux, ou simplement de meilleure qualité architecturale. La socialisation préférentielle est une capitalisation individuelle pour circuler dans plusieurs segments de marchés, elle est aussi une capitalisation collective.

Cette socialisation préférentielle relevait davantage de l'ignorance, de la culpabilisation professionnelle, que de la réalité des pratiques. Dans des marchés difficiles, le jugement porté sur l'architecture produite est alors mis en relation avec les contraintes qui s'y exercent, ce qui ouvre considérablement le potentiel d'intervention. La pratique architecturale légitime ne peut se limiter à quelques productions et marchés ; l'architecte ordinaire, les salariés ou les faux salariés (auto-entrepreneurs), plus nombreux, portent aussi le développement de la profession et la conduit à revoir ses propres critères d'évaluation, tendus jusqu'alors 
vers un absolu, l'équipement public de prestige, et un mode de travail, I'activité projectuelle. En mettant l'accent sur la qualité architecturale, on reste dans un marché sans renier l'originalité de son apport professionnel, plus proche de l'artisan que de l'inventeur de formes. En introduisant plus d'architecture dans l'immobilier de bureau, certains expérimentent des dispositifs constructifs et de gestion du bâti originaux. L'intervention dans l'existant incorpore des conceptions déjà présentes dans le bâti et ravive alors la notion de projet qui tient compte d'un état antérieur sans faire table rase de ce qui précède remettant en cause l'un des principes du Mouvement moderne. En critiquant la pauvreté des compositions urbaines des lotissements de maisons individuelles, les architectes de l'ombre cherchent à produire de la ville à partir de nouvelles densités en pensant que ce travail est aussi essentiel que les grands projets urbains. Néanmoins, des situations de marché génèrent des groupes réfractaires, restés en prise avec la dimension rejetée du marché et dont le travail de conception est trop sous contraintes: les affairistes, les architectes des Bâtiments de France, les salariés de sociétés de promotions. Ainsi des clans, minoritaires, nés d'une morphogenèse disqualifiée, sont bannis.

Sur un autre plan, Bernard Haumont et Jacques Allégret avaient parfaitement identifié les enjeux d'une diversification de la profession et insisté sur l'adéquation entre une division du travail et des trajectoires professionnelles originales d'une partie des étudiants. La programmation et l'urbanisme faisaient partie des ouvertures bénéfiques pour dépoussiérer la profession : le processus s'est renforcé et diversifié, notamment dans le secteur de l'urbanisme, moins du côté de la programmation. La référence à la compétence et à l'adaptation individuelles plutôt que conventionnelles s'est imposée comme un principe majeur de socialisation professionnelle. Le capital individuel et l'accent mis sur les talents originaux deviennent centraux pour s'identifier en tant que professionnel. La formation supérieure y a répondu par une offre pléthorique de formations post-diplômes qui tiennent compte de " niches ", de spécialités nouvelles, d'adaptation à des postes. L'on accorde beaucoup d'importance à la capacité d'expression de soi, aux savoirs singuliers (une thématique, des sites, des institutions, des milieux relationnels) et à l'expérience des individus. Le métier n'est plus seulement la codification par les associations professionnelles, les organisations de travail, mais ce qu'en fait l'individu. La variable individuelle est tout particulièrement effective dans les domaines où des problèmes nouveaux suscitent un appel à des compétences non encore formalisées. C'est le cas du développement durable, du développement économique local, de la concertation-animation, de la gestion immobilière, de la communication et de la médiation. La compétence individuelle est mise en avant et les marchés actuels des expertises favorisent «l'ego » professionnel aux dépens d'un savoir académique, plus formaté.

La diversité des positions, des emplois, des marchés, ne remet pas nécessairement en cause la représentation idéale de l'architecte, capable d'assumer aussi bien le plan de métropoles millionnaires que le design d'un mobilier, des stratégies territoriales que de concevoir une maison écologique. C'est une représentation du 
métier très traditionnelle qui fait sens quand I'aspirant architecte se dirige vers ce type de formation. Le noyau identitaire est la conception et la maîtrise d'œuvre et personne ne le conteste. Mais l'extension vers d'autres domaines d'activités ouvre des perspectives positives, notamment du côté de la production urbaine (programmation, conception). De nombreux architectes, quand ils associent à leur diplôme un parcours de formation orienté vers le projet urbain, voire vers d'autres diplômes, sont recherchés pour leur aptitude au projet, à la projection de scénarios et de solutions, compétences aujourd'hui valorisées. II en va de même pour ceux qui travaillent dans les structures de maîtrise d'ouvrage ou dans des organismes d'études et de planification territoriale, qui peuvent aussi faire reconnaître la dimension spatiale dans des perspectives et des collectifs de travail pluridisciplinaires. Des métiers de la communication et de la médiation culturelle, comme certains la nomment, participent à cette extension, avec une cible : le grand public.

L'hétérogénéité des pratiques est la règle dans une profession et des chercheurs y lisent de la déprofessionnalisation, voire de la déqualification, tandis que d'autres y voient des formes d'adaptation professionnelle indispensables à la modernité. Les réformes récentes du système de formation insistent sur cette diversification et pourtant aucune école d'architecture ne recrutera un étudiant s'il affirme venir pour faire de la programmation, de la communication, de l'expertise ; en revanche, on le recrutera s'il affirme vouloir faire autant du projet urbain de territoire que du projet architectural. II y a vingt ans, cela n'aurait pas été le cas.

\section{La fabrication collective et le projet négocié}

Depuis le début des années 1990, une des avancées théoriques décisives a été de considérer l'architecture comme une production collective, et de souligner I'importance des interactions entre formulation de la commande et solutions spatiales, entre les protagonistes de l'acte de bâtir. Le projet (architectural, urbain, constructif, de territoire, de ville), ses différents états, son identité, s'élaborent dans des itérations successives qui impliquent des négociations à de nombreux niveaux et justifient d'affirmer l'idée de "projet négocié », pour caractériser la manière de fabriquer les espaces bâtis. Les thèses sur l'interprofessionnalité, le forum hybride, I'ingénierie hétérogène ou concourante, la coopération concurrentielle, la distribution des savoirs, ont identifié des règles sur la façon dont les acteurs, décideurs ou professionnels, spécialistes ou profanes, négocient. Dans ces perspectives, la fabrication des espaces se structure sur les liens, les coopérations, les conflits et leur traitement. L'accent mis sur le rôle d'objets intermédiaires (maquettes, images de synthèses, toute représentation graphique), d'investissement de forme (chartes, documents, produits, techniques, procédures, règles de l'art), de contrats, de relations affinitaires ou des conventions, a traduit l'importance d'autres éléments en jeu lors des négociations qui conditionnent les réponses apportées.

Le projet résulte du processus qui le façonne, comme son appropriation et sa réception en dépendent. L'activité de conception est alors replacée dans une chaîne complexe d'action et portée par des acteurs aux intérêts 
pluriels, ce qui oblige à de constants ajustements sur les caractéristiques techniques, formelles, fonctionnelles, symboliques.

La compréhension des échanges et des transactions va au-delà d'approches psychosociologiques ou cognitivistes qui explicitent, dans le cadre d'un colloque singulier, les attitudes, la verbalisation, les comportements, les gestes, qui pèsent sur les choix quels qu'ils soient. La fabrication d'un lieu ou d'une architecture se négocie à un autre niveau quand se mêlent des discours prospectifs, des récits, dit-on aujourd'hui, des savoirs et des compétences singuliers, des procédures pour régler les interventions des acteurs et leurs rôles, un mix qui s'ajuste dans un calendrier mouvant et lui-même enjeu de pressions. En l'occurrence, le projet architectural met en œuvre les politiques publiques ou les stratégies d'acteurs qui le dépassent. La forme architecturale, expression d'un mode de vie et de vivre en collectivité, croise d'autres critères comme le gain politique, la rentabilité de l'investissement public, la recherche de profit chez les privés, la rémunération des acteurs, pour conduire au final à ériger un nouveau morceau de ville et l'architecture qui le représente. Néanmoins, la forme focalise, par la projection imagée et figurative du futur puis par sa matérialité, l'expression des accords entre acteurs et les résultats des arrangements collectifs.

L'analyse en termes de négociation, de dispositifs, de réseaux est une réponse au renouvellement très rapide des conditions de décision, de conception, de gestion des projets, Pratiquement devenu un label pour les législateurs, un instrument d'action pour les collectivités territoriales, un moyen d'insérer la société civile ou l'objet d'une revendication démocratique, une méthode professionnelle pour en optimiser la conception. La consolidation du projet négocié coïncide avec une redéfinition du rôle des acteurs, conjuguée à une accélération de la complexification des modes de transmission de l'information. L'évolution rapide des techniques tant au niveau des outils de conception que des modes constructifs, ou de la communication des projets, ainsi qu'une demande sociale toujours en mouvement, modifient la manière dont les projets sont élaborés, discutés, concertés, négociés. Les bouleversements technologiques et sociaux relayés par les médias et diffusés à l'échelle planétaire ont élargi le champ des acteurs et introduit de nouvelles répartitions des rôles et des compétences.

$\mathrm{Si}$, sur le plan théorique, les chercheurs ont investi les ressorts "du projet négocié », l'enjeu est aussi politique et professionnel : politique, pour intégrer les nombreux acteurs des projets y compris ceux qui pouvaient s'en sentir exclus ; professionnel, car l'importance des négociations modifie l'organisation des procédures et les compétences des experts. Le management a fait son entrée dans un domaine où il semblait réservé aux " grands » projets impliquant des organisations à la fois industrielles et organiques. De plus, dans le champ de l'architecture et de la construction, le projet a été un support d'expérimentation dans de nombreuses opérations, portées par l'État, pour créer ainsi un réservoir significatif d'expériences et de bonnes pratiques. Un secteur innovant s'est structuré sur la technique, sur l'usage, sur les formes, sur l'économie, dans le cadre d'une rénovation des pratiques de projet. 
En particulier, plusieurs programmes de recherche ont travaillé sur la liaison entre programmation-usages et conception-forme : les négociations entre les deux pôles ont fait l'objet d'investigations critiques qui ont contribué à ajuster les procédures d'attribution des marchés ou à faire évoluer les compétences des professionnels.

Dans une logique interprofessionnelle, le milieu doit ajuster la référence à une profession ou à un métier, qui prône des idéaux singuliers, à l'espace professionnel collectif, qui oblige à amender les stratégies de chaque protagoniste. Si le terme de coopération concurrentielle conceptualise un tel paradoxe, l'interprofessionnalité est le signe d'une transformation de la socialisation au travail. L'expérience individuelle, la filiation à une profession princeps, l'appartenance à un espace professionnel pluridisciplinaire en constituent les principaux déterminants. La dimension relationnelle s'affiche avec plus d'intensité à tous les niveaux de la pratique et de l'action.

\section{Conclusion}

Minorer les théories sur la fabrication des espaces au travers d'une analyse en termes d'acteurs et de dispositifs peut apparaître surprenant au regard des effets sur l'architecture et la société. Le déclin de grands récits sur le bien être social, la montée en puissance de l'individualisme, la consommation au cœur des pratiques et des stratégies, perturbent l'œcuménisme d'agir pour le bien collectif dans une mondialisation accrue. Toutes les professions, tous les métiers, toutes les organisations, sont confrontés à l'incertitude sur les finalités, y compris ceux dont on pensait la légitimité sans équivoque (enseignants, médecins, avocats, scientifiques). Phénomènes compensés par les vertus du développement durable, par un renouveau de la pensée ville-nature, par le désir d'offrir à chacun le bien être revendiqué par tous, par l'influence grandissante d'une conscience écologique et environnementale. Loin d'un blanc-seing donné, les savoirs des architectes sont questionnés dans le caractère irréfutable de leurs expertises ou jugements. Les controverses, la médiatisation accrue, l'émergence plus fréquente d'alternatives et de contre-expertises, interrogent l'ambition sociale et démocratique souvent affichée.

Si l'on regarde les architectes sous l'angle de leur mode d'organisation dominant, le terme de "prestataire de service " est en adéquation avec l'univers économique actuel mais leur pose problème. Dans des sociétés individualistes où l'économie et la consommation produisent les principales valeurs, ce registre d'action s'établit autour de la résolution de l'équation marchés-prestations. Dans une vision critique, le compromis avec les valeurs professionnelles (désintéressement, indépendance, valeur collective et publique) est quelquefois difficile à réaliser. Au pire, c'est aller vers une approche mercantile que les architectes récusent, à la différence des grands bureaux d'études, des constructeurs, des entrepreneurs, qui en font leur fond de commerce. C'est être en rupture avec leur idéologie professionnelle et peut être avec leur capacité de développement. Le terme d'expertise suscite moins d'états d'âme car il répond au besoin d'avis qualifiés sur l'architecture. II peut être facilement assumé par les architectes concepteurs, maîtres d'œuvre, et c'est une 
forme de reconnaissance de savoirs acquis et de l'excellence d'une carrière professionnelle (participer à des jurys de concours, conseiller des clients, être sollicité par la presse).

La méconnaissance par de nombreux acteurs et par la population de l'architecture, au sens où l'entendent les experts (le contemporain, le Mouvement moderne par exemple), pénalise durablement un ancrage populaire malgré le désir d'approcher les attentes et les pratiques du plus grand nombre. Demande qui, aux yeux de professionnels, mériterait un traitement égal à des projets emblématiques pour influencer sur la qualité des constructions. Cette faille, si ce n'est faillite professionnelle, maintient un syndrome identitaire qui rend sceptique sur leur compétence. Si l'architecture bénéficie d'une communication (émissions télévisuelles, presse grand public, guides touristiques, sites Internet dédiés), de lieux d'expositions et d'animations certes en nombre limité, elle reste encore une énigme pour beaucoup. Une scène méconnue de diffusion d'une culture architecturale s'est progressivement constituée sous le terme générique de "médiateurs", souvent des architectes. Leur défi est la diffusion de la culture architecturale auprès du " grand public ». II n'est pas facile à relever mais leurs interventions participent à une modernisation du milieu professionnel sous un angle culturel plus qu'économique, ce qui s'avère plus satisfaisant pour beaucoup. Pourtant, les architectes qui viennent à la médiation ont dû franchir des obstacles pour y parvenir et s'affranchir de la représentation dominante. Un métier prend forme, assis sur une solide conviction de devoir communiquer sur l'architecture sans être soi-même un concepteur. La socialisation du plus grand nombre à l'architecture est à ce prix : un ajustement des professions et des métiers. Inversement, l'analyse des métiers renvoie une connaissance de l'architecture. 